\title{
Platelet count and its indices-effectiveness in early diagnosis of neonatal sepsis
}

\author{
Priti Singh, Vipin Chandar \\ From ${ }^{1}$ Visiting Consultant, Department of Pediatrics, BLK-Max Super Speciality Hospital, New Delhi, ${ }^{2}$ Professor and Head, Department of Pediatrics, \\ Himalayan Institute of Medical Sciences, Jolly Grant, Dehradun, Uttarakhand, India
}

\begin{abstract}
Context: Neonatal sepsis is the major cause of morbidity and mortality in developing countries requiring early diagnosis and treatment. The Gold standard Blood culture results are often delayed for $48 \mathrm{~h}$ with high false-negative values. Emerging evidence suggests that platelet indices such as plateletcrit, mean platelet volume (MPV), and platelet distribution width (PDW) are reliable biomarkers that are readily available while obtaining routine complete blood counts. Aim: The aim of the study was to evaluate the efficacy of platelet count (Platelets) and its indices in the early diagnosis of sepsis. Settings and Design: Blood samples were collected from all neonates admitted to the hospital with features of suspected sepsis. Materials and Methods: One hundred patients were recruited for this crosssectional analytical prospective study. All neonates delivered in Himalayan Institute of Medical Sciences and those referred from outside with features of suspected septicemia meeting the inclusion criteria were taken for study purposes. Blood samples were collected at the time of admission. Patients were divided into three groups clinically suspected sepsis (probable sepsis), culture-positive sepsis, and culture-negative sepsis. Non-parametric tests like the Chi-square test were applied to see the association between the variables. The three groups were compared for Platelets and its indices with the Analysis of variance test. Results: MPV was 219.2, 174.8, and $205.7(\mathrm{p}<0.031)$, PDW 8.3, 12.5, and $11.5(\mathrm{p}<0.174)$ and MPV as 10.9, 9.94 and $11.9(\mathrm{p}<0.556)$ in probable sepsis, culture-positive and culture-negative sepsis, respectively. Conclusions: Platelets and its indices can be considered as a diagnostic tool for neonatal sepsis as it is cheap, rapid, and easily available and does not require additional equipment.
\end{abstract}

Key words: Neonatal sepsis, Platelet indices, Thrombocytopenia

$\mathrm{N}$ eonatal sepsis is a diagnosis made in infants $<28$ days of life and consists of a clinical syndrome that may include systemic signs of infection, circulatory shock, and multisystem organ failure [1].

Globally, the Neonatal mortality rate has declined by $47 \%$ between 1990 and 2015 from 36 to 19 deaths per 1000 live births [2]. India contributes to one-fifth of global live births and more than a quarter of neonatal deaths [3]. Neonatal sepsis is one of the major causes of morbidity and mortality among newborns in the developing world and is more common when compared with developed countries [4]. In 2013, the global analysis revealed that the major contributing factors for mortality in neonates were infections and preterm with associated complications in developing countries [3]. Host defense against infection is based on two crucial mechanisms: the inflammatory response and the activation of coagulation. Platelets are involved in both hemostasis and immune response. These mechanisms work together in a complex and synchronous manner making the

\section{Access this article online}

Received - 27 August 2021

Initial Review - 30 December 2021

Accepted - 31 December 2021

DOI: $10.32677 /$ ijch.v9i1.3266 contribution of platelets of major importance in sepsis and platelet count (Platelets) has been used as a marker of sepsis historically.

National Neonatal Forum of India (NNF) has classified sepsis into probable, culture-negative, and culture-positive sepsis [5]. Based on the onset of sepsis, neonatal sepsis is classified into early $(<72 \mathrm{~h}$ ) and late (more than $72 \mathrm{~h}$ ) onset. In early-onset sepsis (EOS), infants acquire infection by a vertical transmission that is through the bacterial flora of the mother. In late-onset sepsis (LOS), the acquisition of infection is predominantly through the infant's environment [6].

Coagulase-negative Staphylococci $(30.27 \%)$ were the most common organisms isolated followed by Acinetobacter sp (15.1\%), Klebsiella sp (5.4\%) Staphylococcus aureus (4.8\%), and Escherichia coli (4.8\%) in a study conducted by Asifa Nazir [7].

For a definite diagnosis of sepsis blood culture has always been the gold standard [8]. However, the blood culture has a huge disadvantage of delay in availability of the result along with other chances of false-negative results [9].

Other tests such as Procalcitonin and IL-6 are very expensive and not easily affordable by most of the patients in developing countries [10]. Hence, there has always been a need for newer

Correspondence to: Dr. Priti Singh, U-142, DLF Capital Green, Motinagar, New Delhi - 110 015, India. E-mail: drpritisingh1532@gmail.com

(C) 2022 Creative Commons Attribution-NonCommercial 4.0 International License (CC BY-NC-ND 4.0). 
innovative methods for early screening of sepsis. Platelets is one of the modalities that is present in routine investigations and is available at an affordable price. In $25-30 \%$ of cases of neonatal sepsis, thrombocytopenia has been seen [11]. Along with Platelets, indices like mean platelet volume (MPV), platelet distribution width (PDW), and plateletcrit (PCT) are also available in coulter. To reduce the neonatal morbidity burden due to sepsis, this study being cheaper, faster, and more accurate was conducted to evaluate the efficacy of Platelets and its indices in septicemia.

\section{MATERIALS AND METHODS}

The study was conducted in the division of Neonatology in the Department of Paediatrics and in the Department of Pathology. The study was conducted in 1 year and written informed consent was taken from the parents. It was a Cross-sectional Analytical study and 100 patients were recruited for the statistical purposes for convenient sampling method.

Neonates delivered in Himalayan Institute of Medical Sciences and referred from outside with features of suspected septicemia or probable sepsis as per NNF criteria of poor feeding, irritability, excessive cry, lethargy poor cry and reflexes, fever, hypothermia, jaundice, vomiting, abdominal distension, tachypnea and grunting, convulsions, diarrhea, pustules, cyanosis, bulge fontanelle, disseminated intravascular coagulation, bleeding, poor perfusion, shock, and apnea were included in the study.

Neonates who developed sepsis during the period of hospitalization for other reasons and proved to have sepsis by positive blood culture, cerebrospinal fluid (CSF), and urine culture were also included in the study while neonates with congenital anomalies of Gastrointestinal Tract system such as trachea-esophageal fistula, malrotation of gut, anomalies of the cardiovascular system or respiratory system, central nervous system, and inborn error of metabolism were excluded from the study.

At admission, thorough history was taken and clinical examination was done. Sepsis profile was sent including hemoglobin, total leukocyte count, differential leukocyte count, Platelets and its indices-MPV, PDW and PCT, absolute neutrophil count (ANC), C-reactive protein (CRP), band cell ratio (BCR), and blood culture for all the patients. BECKMAN COULTER LS-750 Analyzer was used and as when required X-ray chest, Urine $\mathrm{C} / \mathrm{S}$, and $\mathrm{CSF}$ culture was done.

The patients were categorized into three groups-Clinically suspected sepsis (probable sepsis), culture positive sepsis, and culture negative as per NNF criteria of sepsis screen comprising of total leukocyte count, I/T ratio (BCR), ANC, m-ESR e, and CRP $[12,13]$. Statistical Package for the Social Sciences 20.0 version of statistical analysis was used. Sensitivity, Specificity, Positive predictive value (PPV) and Negative predictive value (NPV) of Platelets and its indices were evaluated and compared between probable sepsis with culture negative sepsis and probable sepsis with culture positive sepsis. Non-parametric tests like the Chi-square test were applied to see the association between the variables. The three groups were compared for Platelets and its indices with Analysis of variance (ANOVA) test.

\section{RESULTS}

Sample size of 100 patients was recruited as per hospital neonatal intensive care unit work load during the period of study. The patients were divided into early and late onset. EOS was seen more in males $(64 \%)$ as compared to females $(35.1 \%)$. However, in LOS both male and females were equally affected. On the basis of NNF guidelines, the patients were divided in three groups - 50\% were clinically suspected sepsis (probable sepsis), $34 \%$ were with blood culture positive (culture positive sepsis), and remaining $16 \%$ in blood culture negative but CRP positive group (culture negative sepsis). The general characteristics of a study group showing 100 patients including 61 males and 39 females are shown in Table 1. Out of 40 preterm subjects, 25 (62.5\%) were male and $15(37.5 \%)$ were female whereas out of 60 term subjects $36(60 \%)$ were male and $24(40 \%)$ were females.

Probable sepsis was more among preterm babies whereas culture negative and culture positive were more in term babies.

Sensitivity and accuracy for Platelets were $52.9 \%$ and $58 \%$ in the culture positive group, whereas sensitivity and accuracy for Platelets in the probable sepsis group was $69 \%$ and $57.5 \%$. Specifically, NPV and PPV was $68 \%, 40.7 \%$, and $78.2 \%$ in culture positive whereas it was $68 \%, 21.5 \%$, and $66.6 \%$ in the probable sepsis group (Table 2).

Sensitivity and accuracy for MPV was $26.4 \%$ and $38 \%$ in culture positive and $34 \%, 40.9 \%$ in the probable sepsis group. Specificity, NPV, and PPV were $62.5 \%, 60 \%$, and $38 \%$ in culture positive whereas it was $62.5 \%, 23.2 \%$, and $73.9 \%$ in probable sepsis.

Sensitivity and accuracy of PDW were $14 \%$ and $30 \%$ in culture positive and $28 \%$ and $36 \%$ in probable sepsis. Specificity, NPV, and PPV were $62 \%, 25.6 \%$, and $45 \%$ in culture positive whereas it was $62.5 \%, 21.7 \%$, and $70 \%$ in probable sepsis.

Sensitivity and accuracy of PCT were $17 \%$ and $14 \%$ in culture positive and $86 \%$ and $66.6 \%$ in probable sepsis. Specificity, NPV,

Table 1: Study design of distribution of patients

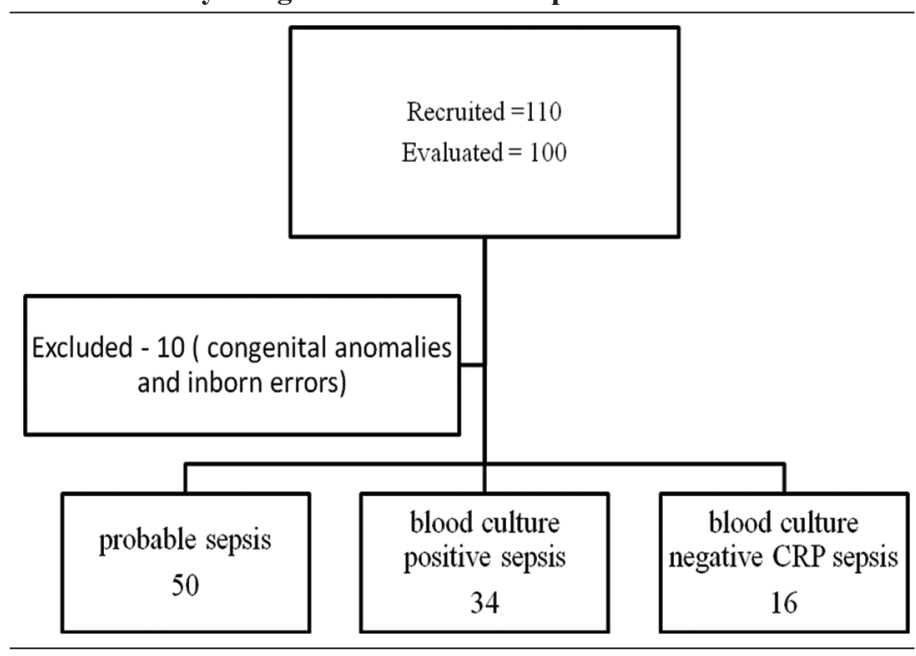


Table 2: Sensitivity and specificity of platelet count and its indices in probable sepsis and culture positive sepsis

\begin{tabular}{|c|c|c|c|c|c|c|c|c|}
\hline \multicolumn{5}{|c|}{ Probable sepsis } & \multicolumn{4}{|c|}{ Culture positive sepsis } \\
\hline Parameters & Platelet count & PDW & MPV & Plateletcrit & Platelet count & PDW & MPV & Plateletcrit \\
\hline Specificity & 68 & 62.5 & 62.5 & 6.2 & 68 & 62 & 62.5 & 6.25 \\
\hline Sensitivity & 69 & 28 & 34 & 86 & 52.9 & 14 & 26.4 & 17 \\
\hline NPV & 21.5 & 21.7 & 23.2 & 12.5 & 40.7 & 25.6 & 28 & 3.4 \\
\hline PPV & 66.6 & 70 & 73.9 & 74.1 & 78.2 & 45 & 60 & 28.5 \\
\hline Accuracy & 57.8 & 36 & 40.9 & 66.6 & 58 & 30 & 38 & 14 \\
\hline
\end{tabular}

PDW: Platelet distribution width, MPV: Mean platelet volume, NPV: Negative predictive value, PPV: Positive predictive value

Table 3: Comparison of normal with probable sepsis and culture positive

\begin{tabular}{|c|c|c|c|c|c|}
\hline Parameters & Normal & Probable sepsis (mean) & Culture $^{+ \text {ve }}$ sepsis (mean) & Culture negative CRP $^{+ \text {ve }}$ (mean) & *p-value \\
\hline Platelet count & $150-400$ & 219.2 & 174.8 & 205.78 & 0.031 \\
\hline PDW & $8.3-56.6 \%$ & 12.5 & 15.1 & 11.05 & 0.174 \\
\hline MPV & $7.2-11.7 \mathrm{fl}$ & 10.9 & 9.94 & 11.9 & 0.556 \\
\hline Platletcrit & $0.22-0.24 \%$ & 2.5 & 1.46 & 3.2 & 0.67 \\
\hline
\end{tabular}

*Chi-square test was applied, PDW: Platelet distribution width, MPV: Mean platelet volume, CRP: C-reactive protein

and PPV was $6.25 \%, 3.4 \%$, and $28.5 \%$, whereas it was $6.2 \%$, $12.5 \%$, and $74.1 \%$ in the probable sepsis group. The receiver operating characteristic curve demonstrates the similar findings (Figs. 1-4).

Analysis on the basis of ANOVA for three groups-culture positive, culture negative, and probable sepsis was done for Platelets and its indices (MPV, PDW, and PCT). Platelets was found to be significant in all groups (Table 3 ).

\section{DISCUSSION}

The need for early recognition, diagnosis of neonatal septicemia, and prompt institution of treatment is paramount to prevent death (s) and complications associated with neonatal septicemia. The definitive diagnosis of sepsis is made by blood culture, which requires a minimum of $48-72 \mathrm{~h}$, yields a positive result in $30-40 \%$ of cases. One of the most common hematological manifestations seen during early sepsis is thrombocytopenia.

Platelet indices; PCT, MPV, and PDW are a group of derived platelet parameters obtained as a part of the automatic complete blood count. PI are biomarkers of platelet activation. Average mean cell volume is 7.2-11.7 fL Increased MPV indicates increased platelet diameter, which can be used as a marker of production rate and platelet activation. During activation, platelets' shapes change from biconcave discs to spherical, and a pronounced pseudopod formation occurs that leads to MPV increase during platelet activation. The PDW reported varies markedly, with reference intervals ranging from 8.3 to $56.6 \%$. Under physiological conditions, there is a direct relationship between MPV and PDW; both usually change in the same direction. PCT is the volume occupied by platelets in the blood as a percentage. The normal range for PCT is $0.22-0.24 \%$.

Platelets play an important role in inflammation. Septic patients are observed to have low Platelets due to production of many cytokines, endothelial damage and bone marrow suppression. PI have been shown to have diagnostic value in certain inflammatory diseases, such as inflammatory bowel

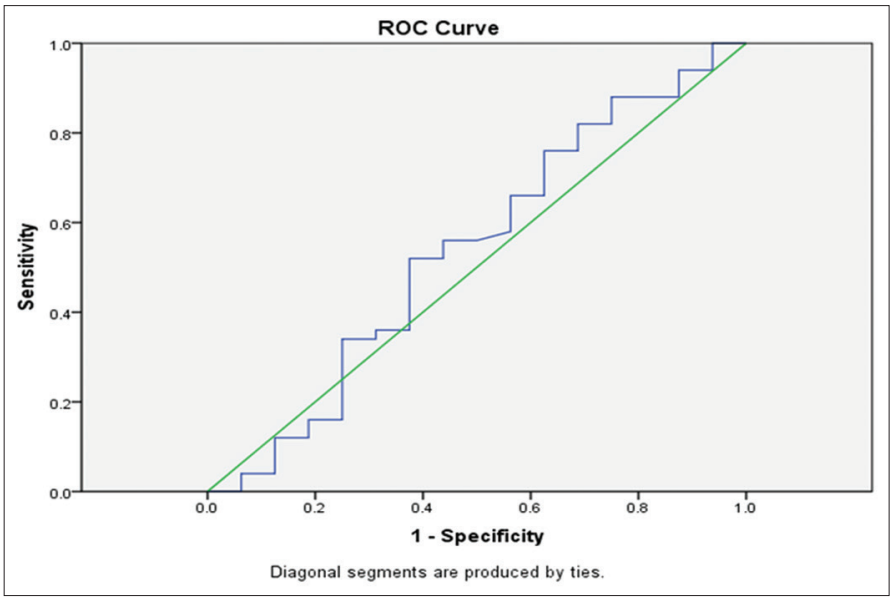

Figure 1: Comparison of platelet count and sepsis

diseases, rheumatoid arthritis, ankylosing spondylitis, ulcerative colitis, and atherosclerosis [14].

In our study, sepsis was predominantly present in males $(61 \%)$ as compared to females (39\%) with early onset sepsis in $64 \%$ and $36 \%$ had late onset sepsis.

In our study, there were $50 \%$ probable sepsis cases, $34 \%$ culture proven cases and 16\% culture negative sepsis. In a similar study conducted by Salama et al. $39.8 \%$ of neonates had probable sepsis and were culture positive [15].

Naik et al. reported thrombocytopenia in $66.25 \%$ of neonates with sepsis having $66.25 \%$ sensitivity and $87 \%$ specificity [16]. Culture positive patients in our study were found to have thrombocytopenia in $72 \%$ of neonates having $52.9 \%$ of sensitivity and $68 \%$ of specificity (Fig. 1) with NPV 40.7\% and PPV 78.2\%. Probable sepsis neonates also had decreased Platelets with sensitivity and specificity as $20 \%$ and $68 \%$, respectively, with NPV of 21.5 and PPV of 6.

In culture positive cases, in our study, PDW sensitivity is $14 \%$ with specificity of $62 \%$ and NPV of 25.6 with PPV of 45 (Fig. 2). While in probable sepsis there was sensitivity of $62 \%$ with specificity of $62.5 \%$ with NPV of 21.7 and PPV of 70 . Sangsari et $a l$. in their study on PI in different types of germs found that 


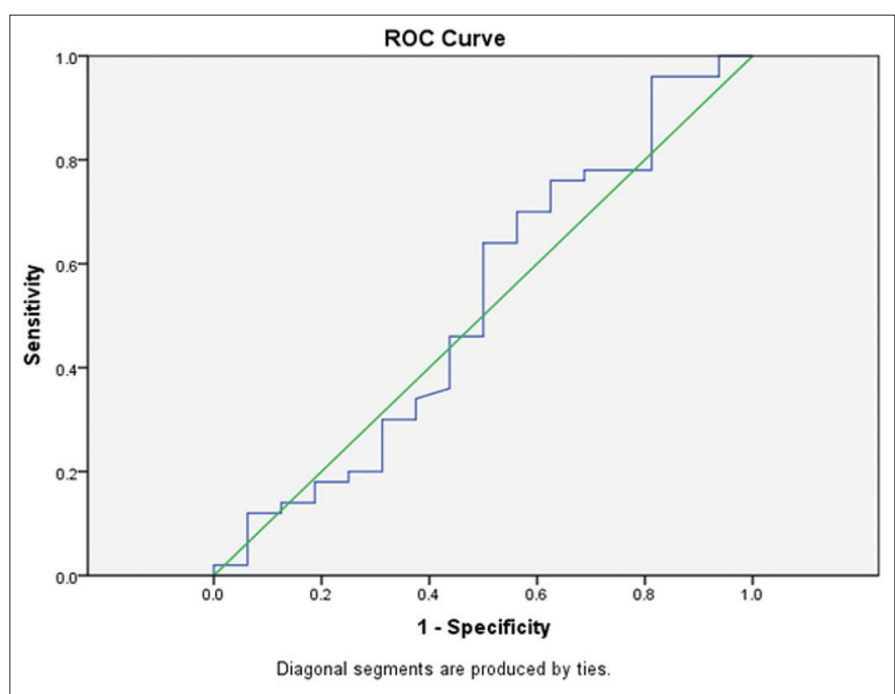

Figure 2: Comparison of Platelet distribution width and sepsis

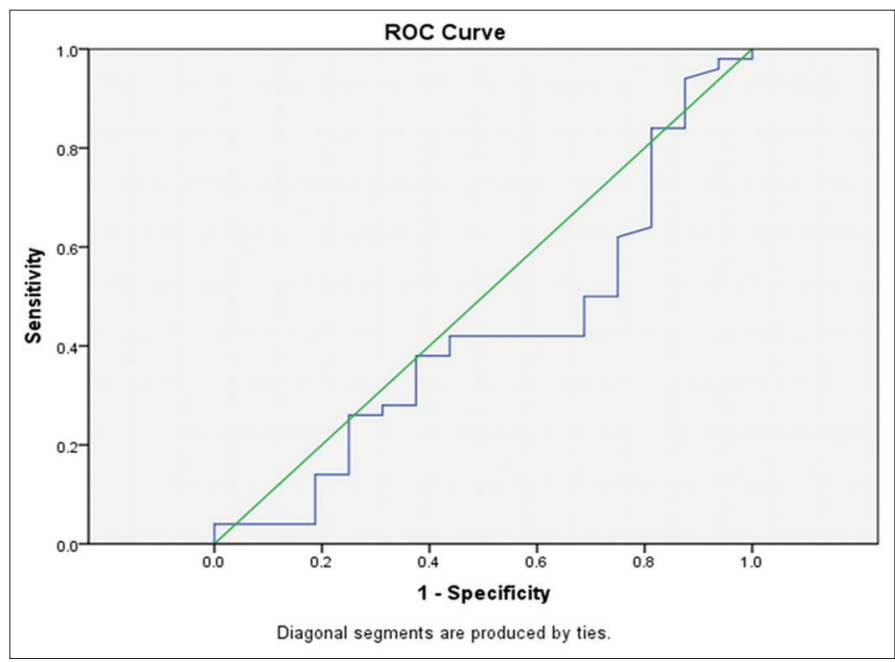

Figure 3: Comparison of Mean platelet volume and sepsis

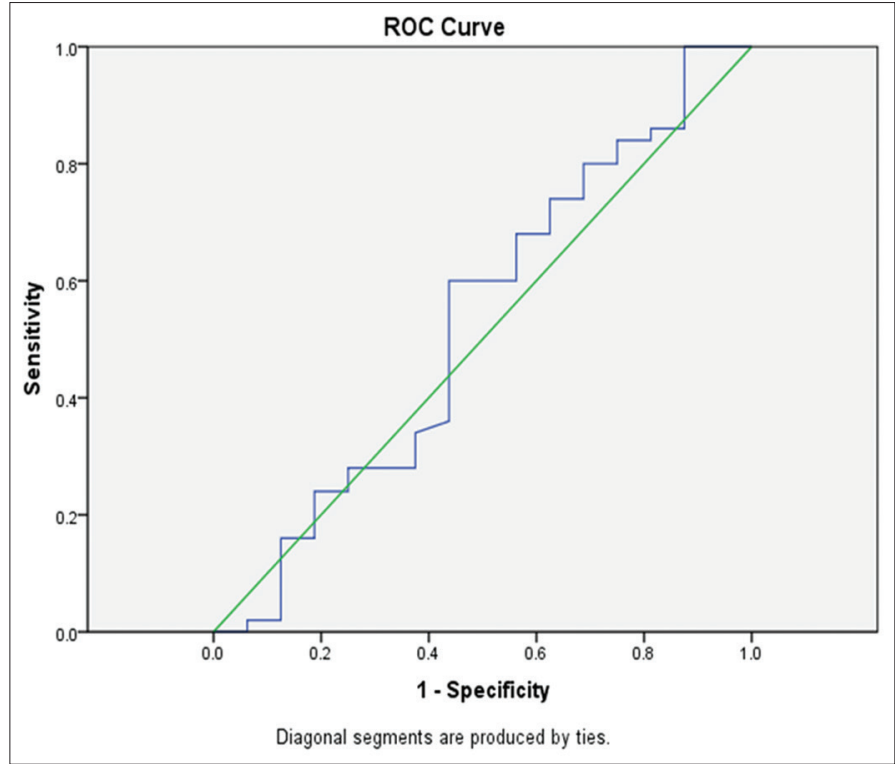

Figure 4: Comparison of Plateletcrit and sepsis

PDW was elevated in all culture positive infections specifically in Gram-negative sepsis [17].
PCT in culture proven cases had sensitivity of $17 \%$ and specificity of $6.25 \%$ with NPV of 3.4 and PPV of 28.5 while in probable sepsis PCT showed sensitivity of $86 \%$ with specificity of $62 \%$ and NPV of 12.5 with PPV of 74.1 (Fig. 4). Very few studies show changes in PCT. Sandeep et al. reported high PDW, low PCT, and high but not significant MPV in preterm neonates which were compared with term neonate (Fig. 3) [18].

The Platelets was significantly low (p value-0.031) in all three groups, probable sepsis, culture positive sepsis and culture negative sepsis; however, the PI such as PDW, MPV, and PCT did not show any significant difference in the various groups.

Platelets may be considered an alternative, novel diagnostic tool for detection of early neonatal sepsis as it is cheap, rapid, and easily available and does not require additional equipment.

Further prospective research is required with increase in sample size to confirm the role of platelet and indices as an early diagnostic tool in neonatal sepsis.

\section{CONCLUSIONS}

Platelets can be considered as an early diagnostic tool for neonatal sepsis as it is cheap, rapid, and easily available and does not require additional equipment.

\section{REFERENCES}

1. Ershad M, Mostafa A, Cruz MD, Vearrier D. Neonatal sepsis. Curr Emerg Hosp Med Rep 2019;7:83-90.

2. Neonatal Mortality; 2021. Available from: https://data.unicef.org/topic/ child-survival/neonatal-mortality. [Last accessed on 20 Nov 2021].

3. Liu L, Oza S, Hogan D, Perin J, Rudan I, Lawn JE, et al. Global, regional, and national causes of child mortality in 2000-13, with projections to inform post-2015 priorities: An updated systematic analysis. Lancet 2015:385:430-40.

4. GHO by Country India Statistics Summary (2002 Present); 2017. Available from: http://apps.who.int/gho/data/node.country.country-IND [Last accessed on 20 Jul 2017].

5. Klingenberg C, Kornelisse RF, Buonocore G, Maier RF, Stocker M. Culturenegative early-onset neonatal sepsis at the crossroad between efficient sepsis care and antimicrobial stewardship. Front Pediatr 2018;6:285.

6. Richard J, Fanaroff AA, Walsh M. Postnatal Bacterial infections. In: Ethan L, Dobbs K, editors. Fanaroff and Martin's Neonatal Perinatal Medicine. $10^{\text {th }}$ ed. Philadelphia, PA: Elsevier Health Sciences; 2015. p. 734-50.

7. Ghotaslou R, Ghorashi Z, Nahaei MR. Klebsiella pneumoniae in neonatal sepsis: A 3-year-study in the pediatric hospital of Tabriz, Iran. Jpn J Infect Dis 2007;60:126-8.

8. Sonawane VB, Mehkarkar N, Gaikwad S, Kadam N. Comparison between sepsis markers and blood culture in diagnosis of neonatal sepsis: A prospective study. Int J Res Med Sci 2017;5:1662-6.

9. Benitz WE. Adjunct laboratory tests in the diagnosis of early-onset neonatal sepsis. Clin Perinatol 2010;37:421-38.

10. Ku LC, Boggess KA, Cohen-Wolkowiez M. Bacterial meningitis in infants. Clin Perinatol 2015;42:29.

11. Bhat YR. Platelet indices in neonatal sepsis: A review. World J Clin Infect Dis 2017;7:6-10.

12. Polinski $\mathrm{C}$. The value of the white blood cell count and differential in the prediction of neonatal sepsis. Neonatal Netw 1996;15:13-23.

13. Da Silva O, Ohlsson A, Kenyon C. Accuracy of leukocyte indices and C-reactive protein for diagnosis of neonatal sepsis: A critical review. Pediatr Infect Dis J 1995; 14:362-6.

14. Margetic S. Inflammation and haemostasis. Biochem Med (Zagreb) 
2012;22:49-62.

15. Salama K, Gad A, El Tatawy S. Sepsis profile and outcome of preterm neonates admitted to neonatal intensive care unit of Cairo University Hospital. Egypt Pediatr Assoc Gaz 2021;69:8.

16. Bhat S, Naik S, Rafiq W, Tariq AS. Incidence of thrombocytopenia and changes in various platelet parameters, in blood culture positive neonatal sepsis. Int J Pediatr 2015;3:757-66.

17. Sangsari R, Kadivar M, Fardi R, Attary SJ, Jafari A. Platelets indices and different germs of neonatal sepsis. Iran J Pediatr 2019;29:e80856.
18. Sandeep M, Thammanna PS, Sridhar PV. Platelet indices in preterm neonates: A prospective study. Int J Sci Stud 2015;3:237-40.

Funding: None; Conflicts of Interest: None Stated.

How to cite this article: Singh $\mathrm{P}$, Chandar V. Platelet count and its indices-effectiveness in early diagnosis of neonatal sepsis. Indian $\mathrm{J}$ Child Health. 2022; 9(1):1-5. 